\title{
New species and combinations in Helvella and Gyromitra
}

\author{
HARRI HARMAJA
}

\begin{abstract}
HARMAJA, H. 1978: New species and combinations in Helvella and Gyromitra. Karstenia 18: 57.

Descriptions are given of the following new cupulate species of Helvella St.-Am. (Pezizales): $H$. hyperborea Harmaja (type from Finland), $H$. oblongispora Harmaja (type from the Federal Republic of Germany), and $H$. pedunculata Harmaja (type from Finland). Geopyxis verruculosa Sacc. (Peziza verruculosa Berk. \& Curt. non al.) is transferred to Helvella as $H$. verruculosa (Sacc.) Harmaja, n. comb. Paxina recurvum Snyder is transferred to Gyromitra Fr. as G. recurva (Snyder) Harmaja, n. comb.
\end{abstract}

Harri Harmaja, Botanical Museum, University of Helsinki, Unioninkatu 44, SF00170 Helsinki 17, Finland

Three new cupulate species and two new combinations in the genera Helvella St.-Am. and Gyromitra Fr. (Pezizales) are published here, partly to permit their inclusion in the Nordic Flora of Macromycetes now under preparation. More detailed accounts of all the species will be published in forthcoming papers.

Helvella hyperborea Harmaja, n. sp. - Helvellae costiferae valde propinqua. Ab ea differt apotheciis plerumque obscurioribus, costis apothecii minus prominentibus non vel parce ramosis, superficie exteriore apothecii brunneopubescenti, paraphysibus dissimilibus, cellulis texturae prismaticae brunneoincrustatis et distributione hyperborea. Typus: Finland, prov. Kuusamo, par. Kuusamo, Juuma, western part of the gorge Jäkälävuoma, alt. ca. $205 \mathrm{~m}$, on a shady shelf in the basal part of a steep dolomitic rock, among the moss Distichium capillaceum (etc.), accompanied by Salix reticulata, Saxifraga aizoides, S. nivalis, Woodsia glabella, Gerronema albidum, 27. VIII.1970 H. Harmaja (H). Specimens also seen from Sweden, Norway, and Iceland.

Helvella oblongispora Harmaja, n. sp. - Helvellae leucomelaenae sat similis; $a b$ ea differt coloribus pallidioribus et stipite costis satis distinctis apothecii, constructione excipuli dissimili et sporis brevioribus (longitudine 17.5-21.0 $\mu \mathrm{m}$ ) plerumque oblongis. Typus: Federal Republic of Germany, Bavaria (Bayern), near Munich (München), abundant on calcareous soil under conifers near the River Isar, 23.VII. 1969 A. Einhellinger (C). Specimens also seen from Sweden, Norway, and Austria.

Helvella pedunculata Harmaja, n. sp. - $A$ Helvella leucomelaena praecipue differt apotheciis tenuiter pedunculatis stipite costato et sporis brevioribus (longitudine ca. 17.5-20.0 $\mu \mathrm{m}$ ). Typus: Finland, prov. Pohjois-Pohjanmaa, par. Kiiminki, Keskikylä limestone area, $\mathrm{N}$ end of Isohalmeenmaa, $50 \mathrm{~m} \mathrm{~W}$ of the spring, in rich fen among moss, 2.VII.1975 E. \& M. Ohenoja (OULU). Specimens also seen from Norway.

Helvella verruculosa (Sacc.) Harmaja, n. comb. (Geopyxis verruculosa Sacc., Sylloge fungorum 8: 68. 1889; Peziza verruculosa Berk. \& Curt., Proc. American Acad. Arts Sci. 4: 127. 1860; Isotype (FH) examined. - Non Peziza verruculosa Weinm., Sylloge plantarum novarum 2: 111. 1827; nec Peziza verruculosa Berk. \& Broome, Journ. Linnean Soc. London 14: 105. 1875.)

Gyromitra recurva (Snyder) Harmaja, n. comb. (Paxina recurvum Snyder, Mycologia 28: 487. 1936. - Type material (NY) examined.) 